\title{
Better Selection Model for EML4-ALK Fusion Gene Test in Patients with Non-Small-Cell Lung Cancer*
}

\author{
Dekel Shlomi ${ }^{1}$, Amir Onn ${ }^{1}$, Maya Gottfried ${ }^{2}$, Jair Bar ${ }^{1}$, Haim Biran ${ }^{1}$, Maya Ilouze ${ }^{1}$, Addie Dvir ${ }^{3}$, \\ Hovav Nechushtan ${ }^{4}$, Lior Soussan-Gutman ${ }^{3}$, Nir Peled ${ }^{1 \#}$ \\ ${ }^{1}$ Pulmonary-Oncology Unit, Chaim Sheba Medical Center, Tel Hashomer, Israel; ${ }^{2}$ Oncology Department, Meir Medical Center, Kfar \\ Saba, Israel; ${ }^{3}$ Oncotest-TEVA, Teva Pharmaceutical Industries LTD., Shoham, Israel; ${ }^{4}$ Sharett Institute of Oncology, Hadassah He- \\ brew University Medical Center, Jerusalem, Israel. \\ Email: "nirp@post.tau.ac.il
}

Received July $5^{\text {th }}$, 2013; revised August $6^{\text {th }}, 2013$; accepted August $14^{\text {th }}, 2013$

Copyright (C) 2013 Dekel Shlomi et al. This is an open access article distributed under the Creative Commons Attribution License, which permits unrestricted use, distribution, and reproduction in any medium, provided the original work is properly cited.

\begin{abstract}
Background: In the last decade, the search for gene mutations in lung cancer has been constantly growing. EGFR, KRAS mutations and, recently, the EML4-ALK fusion can guide the selection of treatment for patients who carry a specific mutation. Methods: During 2010-2011, EML4-ALK fusion test has been performed in Israel, mostly for wild type EGFR non-squamous NSCLC patients based on fluorescent in-situ hybridization (FISH) technique to detect EML4-ALK rearrangements. Results: Between January 2010 and December 2011, 3341 patients were diagnosed with lung cancer in Israel. Of the 2997 patients with NSCLC 687 had squamous cell carcinoma and 2310 had non-squamous NSCLC. This study focused on available 125 non-squamous NSCLC cases in which analysis for EML4-ALK rearrangement was available. All were negative for EGFR mutation. Nineteen (15.2\%) were found positive for the fusion, a figure 2 - 10 times higher compared with previously reported findings. The EML4-ALK fusion was significantly more prevalent in younger male patients (52.1 vs. 61.3 years, $p=0.049$ ), in whom every additional year reduced the chance to find the fusion by $7 \%[\mathrm{CI}=0.93(0.88-0.99), \mathrm{p}=0.03]$. Conclusions: A stepwise approach based on histology and prior EGFR analysis to detect EML4-ALK fusion is highly efficient with a related increased yield of detection. We recommend testing patients with non-squamous cell lung carcinoma after ruling out an EGFR mutation. The chance to find the ALK fusion is significantly greater in younger men.
\end{abstract}

Keywords: Lung Cancer; EML4-ALK; Gene Mutation; EGFR; Histology

\section{Introduction}

In the past few years, a novel therapeutic approach to lung cancer treatment has been integrated into clinical practice. The epidermal growth factor receptor 1 (HER1/ EGFR) inhibitors, such as Erlotinib (Tarceva, Genentech, USA) and Gefitinib (Iressa, AstraZeneca, UK), were the first two oral drugs to demonstrate efficacy in patients who harbored an activating mutation in the EGFR gene $[1,2]$. Searching for other gene mutations as possible therapeutic targets, Soda et al. identified, in 2007, a unique rearrangement of the anaplastic lymphoma kinase

\footnotetext{
*Jair Bar received honoraria and reimbursement for the attendance of a scientific conference from Pfizer. Hovav Nechushtan participated in phase IV Sutent study by Pfizer. Lior Soussan-Gutman is an employee of "Teva Pharmaceutical Industries” LTD. All other authors declared no conflict of interests.

${ }^{\#}$ Corresponding author.
}

(ALK) gene in 6.7\% (5/75) Japanese patients with nonsmall-cell lung cancer (NSCLC) who were negative for the EGFR mutation [3]. In this study, the ALK rearrangement was described as a small inversion within chromosome $2 \mathrm{p}$, resulting in the formation of a fusion gene comprising portions of the echinoderm microtubuleassociated protein-like 4 (EML4) gene and the ALK gene. The ALK gene is a target for research and has been targeted in anaplastic large cell lymphoma [4] and inflammatory myofibroblastic tumor (IMT) [4,5]. In lung cancer, the EML4-ALK fusion gene was found almost exclusively in wild-type EGFR and KRAS adenocarcinomas [6-10]. Similar results were reported by Wong et al., although two out of the 13 ALK positive tumors were described as comprised of mixed squamous and glandular components [8]. The discovery of EML4-ALK fusion as a therapeutic target in lung cancer led to the develop- 
ment of an ALK tyrosine-kinase inhibitor (TKI) Crizotinib (Xalkori, Pfizer, USA), for advanced NSCLC patients who harbor this mutation [11].

This article summarizes our experience in identifying EML4-ALK fusion in NSCLC patients, in the Israeli population during the period 2010-2011.

\section{Materials and Methods}

This study summarized our experience with EML4-ALK analysis performed from January 2010 to December 2011. The study was approved by an inter-institutional review board. The analysis has been performed mostly to wild type EGFR NSCLC patients with non-squamous cell histology. Patients with insufficient tissue for genetic testing, or for whom EML4-ALK fluorescent in-situ hybridization (FISH) was inconclusive, were excluded. When the tumor area had been determined on the hematoxylin and eosin (H\&E) slide from a formalin-fixed paraffin-embedded (FFPE) patient specimen, an ALK gene rearrangement was assessed utilizing the Vysis ALK break-apart FISH kit (Abbott Molecular). Identification probes for LSI ALK 5' probe (Spectrum Green) and LSI ALK 3' probes (Spectrum Orange) were applied, hybridized, and assessed along with standard controls. At least 50 non-overlapping nuclei were analyzed, and the localization of the LSI ALK 5' probe (green) and LSI ALK 3' probe (orange) signals were recorded and interpreted according to manufacturer's guidelines. A positive result for a $A L K$ rearrangement were considered if $15 \%$ of cells or more had split 3' $A L K$ and 5' $A L K$ signals apart by $>2$ times the signal size or a single 3' $A L K$ signal (Figure 1).

Statistical analysis: Student's t-test and chi-square test were used to examine associations between the ALK gene rearrangement and independent covariates. Unconditional logistic regression was used to assess the odds of having an ALK gene rearrangement with respect to risk factors (such as sex and age) and risks were expressed in terms of odds ratios and respective $95 \%$ confidence intervals.

\section{Results}

Between January 2010 and December 2011 lung cancer was diagnosed in 3341 patients in Israel. Among them, 344 patients had SCLC and 2997 had NSCLC. In the NSCLC group 687 had squamous cell carcinoma and 2310 had non-squamous NSCLC. In this study we focused on 125 NSCLC patients whom their EML4-ALK analysis was available (Table 1). EML4-ALK fusion was detected in 19 patients (15.2\%), of whom 10 were men and 9 women. The average age of the ALK positive group was 56.4 years, non-significantly lower than the average age of 61.3 years of the ALK negative patients $(p=0.14)$. However, when age was stratified according to

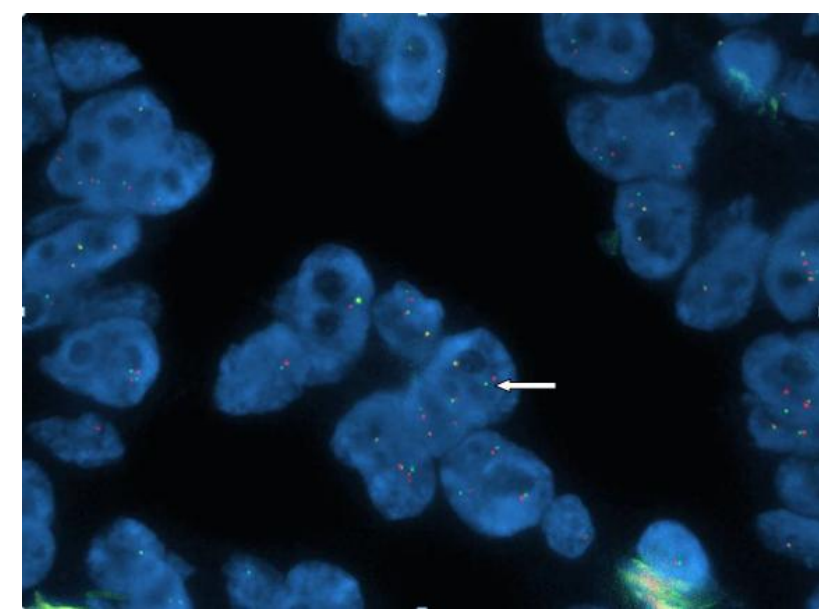

Figure 1. FISH assay in NSCLC patient positive for EML4ALK fusion. Result were considered positive if more than 15\% of cells had split 3' ALK (Spectrum Orange) and 5' $A L K$ (Spectrum Green) signals apart by $>2$ times signal size or single 3' $A L K$ signal (arrow) in at least 50 non-overlapping nuclei.

Table 1. Clinical characteristic of patients with EML4-ALK fusion test in non-squamous, EGFR negative, NSCLC patients in Israel, during 2010-2011.

\begin{tabular}{ccccc}
\hline Characteristic & ALL & ALK+ & ALK- & p-value \\
\hline No. of patients & 125 & $19(15.2 \%)$ & $106(84.8 \%)$ & \\
Sex & & & & \\
Male & 60 & $10(52.6 \%)$ & $50(47.2 \%)$ & 0.66 \\
Female & 65 & $9(47.4 \%)$ & $56(52.8 \%)$ & 0.14 \\
$\begin{array}{c}\text { Age (yrs) } \\
\text { Mean } \pm \text { SD }\end{array}$ & & $56.4 \pm 12.9$ & $61.3 \pm 11.9$ & 0.049 \\
Male & & $52.1 \pm 13.2$ & $61.96 \pm 12.1$ & 0.9 \\
Female & & $61.2 \pm 11.4$ & $60.7 \pm 11.8$ & \\
Ever smoked & & & & \\
No & $6(66.7 \%)$ & $27(40.3 \%)$ & \\
Yes & $3(33.3 \%)$ & $40(59.7 \%)$ & 0.13 \\
Unknown & 10 & 39 & \\
\hline
\end{tabular}

EGFR: epidermal growth factor receptor.

sex, we found the ALK fusion to be more common in younger men; 52.1 vs. 62 years of age $(p=0.049)$. When unconditional logistic regression was used to assess the odds of having the ALK fusion with respect to risk factors, we found that for men, every additional year lessens the chance to find the fusion by $7 \%$ [CI $=0.93(0.88-$ 0.99), $p=0.03$ ]. Smoking information was available for 9 ALK positive patients, in whom 6 (66.7\%) were lifetime never smokers. In the ALK negative patients group, smoking information was available for 67 patients, in whom 27 (40.3\%) were lifetime never smokers. There 
were no statistical significant differences regarding smoking habits between the ALK positive and the ALK negative patients $(p=0.13)$. When the origin of the biopsy was tested for ALK fusion, we found no correlation between biopsies that were taken from the lung origin or the metastasis.

\section{Discussion}

While EGFR mutation is prevalent in 15\% - 20\% of NSCLC patients, being more common in Asian ethnicity, women and non-smokers [1,12-14], the EML4-ALK fusion is less prevalent, more common in Asian population, younger and non-smokers or light smokers. To date, no conclusive data was published regarding sex-depended differences.

Ethnic variation of the EML4-ALK fusion gene shows a frequency as high as 7.5\% (9 of 120) of NSCLC specimens from Italy and Spain [15], while in US, Koivunen et al. found a frequency of 1.5\% (2 of 138 ) in US Caucasian population [16]. In this study, Korean patients had a non-significant 3.6\% (total population 3\%) more prevalence of the EML4-ALK fusion. In Japan, Soda et al. [3] detected the EML4-ALK fusion in $6.7 \%$ of the NSCLC patients while Inamura et al. [6] detected ALK rearrangement in $2.26 \%$ (5 of 221) patients with primary lung cancer. In Chinese population, 4.9\% (13/266) were positive for EML4-ALK fusion, in the study of Wong et al. of NSCLC patients [8].

Histology: In this study, patients were selected to the EML4-ALK fusion test if they had non-squamous lung carcinoma and wild type EGFR gene analysis. To the best of our knowledge, all studies except one found ALK fusion in non-squamous lung carcinomas. Inamura et al. found EML4-ALK fusion in 11 of 363 in primary lung cancer, all adenocarcinomas of papillary or acinar type [7]. Similarly, all of the EML4-ALK fusion-containing tumors in both Koivunen et al. [16] and Takahashi [10] studies were adenocarcinomas. In Shaw et al., 18 of 19 EML4-ALK fusion tumors were adenocarcinoma, predominantly of the signet ring cell subtype, and one was of the adenosquamous type, however this result could be due to selection bias [9]. In Chinese population, 11 of the EML4-ALK fusion were adenocarcinomas, and 2 were unusual carcinomas with mixed squamous and glandular components [8]. The most outstanding results were found in the Martelli et al. study in Italy, in which, of the 9 NCSLC patients with EML4-ALK fusion, 3 were found to be adenocarcinoma, 2 adenosquamous carcinoma and 4 squamous cell carcinomas [15].

EGFR and KRAS mutations: Several studies found the EML4-ALK fusion exclusively in wild-type EGFR and KRAS tumors in NSCLC patients [6-10,17]. Only one of 8 patients with EML4-ALK fusion adenocarcinoma of the lung, in the Koivunen et al. study, was positive for EGFR mutation, while all of them were negative for KRAS mutation [16]. In the Martelli et al. study, all of the 9 patients with EML4-ALK fusion lung cancer were negative for EGFR mutation, but KRAS mutation was detected in one patient with lung adenocarcinoma [15]. Based on this data, ALK fusion testing in Israel is focused on patients with non-squamous lung carcinoma who were negative for the EGFR mutation. By selecting NSCLC patients with non-squamous lung carcinoma and negative EGFR mutation, we could expect a higher prevalence of the EML4-ALK fusion and were thus hoping to cut on unnecessary high cost tests. Accordingly, out of the tested population in our study a frequency of $15.2 \%$ of EML4-ALK fusion was found, which is $2-10$ times higher than previously reported $[6,8,15,16]$.

Age: We found a non-significant lower age for patients in the EML4-ALK fusion group (56.4 vs. 61.3 years, $\mathrm{p}=$ 0.14). However, mean age of males with positive results was 9.86 years younger than males with negative result (52.1 vs. 61.96 years, $p=0.049$ ). We also found that among men, every additional year reduced the chance to find the fusion by $7 \%$, which was statistically significant [CI $=0.93$ (0.88 - 0.99), $\mathrm{p}=0.03$ ]. These findings were a further support to previous studies that indicated a statistically significant prevalence of ALK fusion in younger patients [7-9,17]. In the Wong et al. study, the ALK positive group had median age of 59 years, compared with the ALK negative patients, in whom the median age was $64(\mathrm{p}=0.018)$ [8]. Inamura et al. found that patients with EML4-ALK lung cancers were younger (mean age 56 vs. 64 years, $p=0.0062$ ) [7]. Four of 11 patients $(36 \%)$ with EML4-ALK-positive lung cancers were below 50 years of age, as compared with 12 of 242 patients (5.0\%) with EML4-ALK-negative lung cancers ( $p=0.00038)$. In the Shaw study, patients with EML4-ALK fusion tumors were of a significantly younger median age of 52 compared with 66 years of age in patients with EGFR mutation and 64 years of age in patients with WT/WT status [9]. However, in the Martelli et al. study, no age correlation was noted [15].

Sex: Previous studies found mixed results in regard to sex differences. However, this study showed no sex preferences for the EML4-ALK fusion (10 males vs. 9 females, $\mathrm{p}=0.66)$. In Japan, 4 of the 5 patients who were positive for the EML4-ALK fusion genes were females [10]. In a combined US and Korean population study, the frequency of EML4-ALK fusion was non-significantly higher in females (4\%) versus males (2\%) [16]. The study of Shaw et al. in US, mostly of non-Asian population, identified a significantly greater prevalence of EML4-ALK fusion in men (23\%) than in women (9\%) [9], while Martelli et al. did not find any correlation with 
sex [15].

Smoking: Our data collection was not comprehensive enough to determine smoking habits differences among groups. Smoking exposure data was collected from 76 of 125 patients $(60.8 \%)$, but no statistically significant differences were found among the groups. Several studies showed that the EML4-ALK fusion was more frequent in never smokers or light smokers [8-10,16-17]. In the Koivunen et al. study, the EML4-ALK fusion was detected significantly more frequently in patients with limited smoking history ( $<10$ pack-years) compared with tumors from smokers [16]. In the Shaw et al. study [9], patients who were positive for EML4-ALK fusion were more likely to be never smokers or light smokers, compared with patients in the wild-type cohort $(\mathrm{p}<0.001)$. Also in the Wong et al. study, the EML4-ALK fusion was significantly higher in non-smokers [8]. In the Takahashi et al. study, the EML4-ALK fusion was predominantly found in non-smokers or light smokers [10]. Other studies found no association between EML4-ALK fusion and smoking habits $[7,15]$.

\section{Conclusion}

EML4-ALK fusion in selected patients in Israel with non-squamous cell lung carcinoma, in whom the EGFR mutation test was negative, was found to be more frequent (15.2\%) than expected when no selection was introduced. In this group, the ALK fusion was significantly more prevalent in younger men. Based on this study and on published literature, in order to avoid unnecessary ALK fusion tests, we recommend selecting patients with non-squamous cell lung carcinoma in whom both the EGFR and the KRAS mutations are negative.

\section{Acknowledgements}

The authors thank Oncotest-TEVA Pharmaceutical industries LTD. for their assistance in data management.

\section{REFERENCES}

[1] T. J. Lynch, D. W. Bell, R. Sordella, et al., "Activating Mutations in the Epidermal Growth Factor Receptor Underlying Responsiveness of Non-Small-Cell Lung Cancer to Gefitinib," New England Journal of Medicine, Vol. 350, No. 21, 2004, pp. 2129-2139.

[2] F. A. Shepherd, J. R. Pereira, T. Ciuleanu, E. H. Tan, V. Hirsh, S. Thongprasert, et al., "Erlotinib in Previously Treated Non-Small-Cell Lung Cancer," New England Journal of Medicine, Vol. 353, 2005, pp. 123-132. doi:10.1056/NEJMoa050753

[3] M. Soda, Y. L. Choi, M. Enomoto, et al., "Identification of the Transforming EML4-ALK Fusion Gene in NonSmall-Cell Lung Cancer," Nature, Vol. 448, No. 7153, 2007, pp. 561-566.
[4] M. Shiota, S. Nakamura, R. Ichinohasama, et al., “Anaplastic Large Cell Lymphomas Expressing the Novel Chimeric Protein p80NPM/ALK: A Distinct Clinicopathologic Entity,” Blood, Vol. 86, No. 5, 1995, pp. 1954-1960.

[5] J. Cools, I. Wlodarska, R. Somers, et al., "Identification of Novel Fusion Partners of ALK, the Anaplastic Lymphoma Kinase, in Anaplastic Large-Cell Lymphoma and Inflammatory Myofibroblastic Tumour," Genes Chromosomes Cancer, Vol. 34, No. 4, 2002, pp. 354-362.

[6] K. Inamura, K. Takeuchi, Y. Togashi, et al., "EML4ALK Fusion Is Linked to Histological Characteristics in a Subset of Lung Cancers,” Journal of Thoracic Oncology, Vol. 3, No. 1, 2008, pp. 13-17.

[7] K. Inamura, K. Takeuchi, Y. Togashi, et al., "EML4ALK Lung Cancers Are Characterized by Rare Other Mutations, a TTF-1 Cell Lineage, an Acinar Histology, and Young Onset," Modern Pathology, Vol. 22, No. 4, 2009, pp. 508-515. doi:10.1038/modpathol.2009.2

[8] D. W. Wong, E. L. Leung, K. K. So, et al., "The EML4ALK Fusion Gene Is Involved in Various Histologic Types of Lung Cancers from Non-Smokers with WildType EGFR and KRAS,” Cancer, Vol. 115, No. 8, 2009, pp. 1723-1733. doi:10.1002/cncr.24181

[9] A. T. Shaw, B. Y. Yeap, M. Mino-Kenudson, et al., "Clinical Features and Outcome of Patients with NonSmall-Cell Lung Cancer Who Harbor EML4-ALK," Journal of Clinical Oncology, Vol. 27, No. 26, 2009, pp. 4247-4253. doi:10.1200/JCO.2009.22.6993

[10] T. Takahashi, M. Sonobe, M. Kobayashi, et al., "Clinicopathologic Features of Non-Small-Cell Lung Cancer with EML4-ALK Fusion Gene,” Annals of Surgical Oncology, Vol. 17, No. 3, 2010, pp. 889-897.

[11] A. T. Shaw, B. Y. Yeap, B. J. Solomon, et al., "Effect of Crizotinib on Overall Survival in Patients with Advanced Non-Small-Cell Lung Cancer Harbouring ALK Gene Rearrangement: A Retrospective Analysis,” Lancet Oncology, Vol. 12, No. 11, 2011, pp. 1004-1012.

[12] R Development Core Team, "R: A Language and Environment for Statistical Computing," R Foundation for Statistical Computing, Vienna, 2006.

http://www.R-project.org

[13] H. Shigematsu, L. Lin, T. Takahashi, et al., "Clinical and Biological Features Associated with Epidermal Growth Factor Receptor Gene Mutations in Lung Cancers," Journal of the National Cancer Institute, Vol. 97, No. 5, 2005, pp. 339-346. doi:10.1093/jnci/dji055

[14] R. Rosell, T. Moran, C. Queralt, et al., "Screening for Epidermal Growth Factor Receptor Mutations in Lung Cancer," New England Journal of Medicine, Vol. 361, 2009, pp. 958-967. doi:10.1056/NEJMoa0904554

[15] M. P. Martelli, G. Sozzi, L. Hernandez, et al., "EML4ALK Rearrangement in Non-Small-Cell Lung Cancer and Non-Tumor Lung Tissues," American Journal of Pathology, Vol. 174, No. 2, 2009, pp. 661-670.

[16] J. P. Koivunen, C. Mermel, C. Murphy, et al., "EML4ALK Fusion Gene and Efficacy of an ALK Kinase Inhibitor in Lung Cancer," Clinical Cancer Research, Vol. 14, No. 13, 2008, pp. 4275-4283. 
[17] S. J. Rodig, M. Mino-Kenudson, S. Dacic, et al., "Unique Clinicopathologic Features Characterize ALK-Rearranged Lung Adenocarcinoma in the Western Population,” Cli- nical Cancer Research, Vol. 15, No. 16, 2009, pp. 52165223. 